\title{
MORTALIDAD MATERNA COMPARATIVA ENTRE DOS PERIODOS 1963-1967 1968-1972
}

\author{
Dr. Alfonso Jubiz Hasbún* \\ Dra. Carmen C. Sánchez M.** \\ UNIVERSIDAD DE ANTIOQUIA - FACULTAD DE MEDICINA \\ DEPARTAMENTO DE OBSTETRICIA Y GINECOLOGIA \\ HOSPITAL UNIVERSITARIO SAN VICENTE DE PAUL - Medellín, Colombia - 1975
}

\section{Introducción :}

Los estudios de mortalidad materna y perinatal son indicadores válidos para medir la calidad de la atención que se brinda al binomio madrefeto. Las causas de muertes maternas pueden variar de una ciudad a otra y dependen de muchos factores, tales como la edad y paridad, el tipo de atención obstétrica que se brinde y el nivel socioeconómico. Las tasas de mortalidad materna pueden ser muy bajas como las de San Francisco 1.1 y $\tan$ elevadas como las de Cali 33.7 y 40.9 en Santiago de Chile (7).

Es importante anotar que las tasas de mortalidad materna han disminuído como consecuencia de los adelantos en la atención obstétrica, sin embargo, continúan elevadas en países en vías de desarrollo y se acepta que el $75 \%$ de las muertes maternas son debidas a hemorragia, toxemia e infección y casi el $90 \%$ de ellas son susceptibles de prevención (7).

Entre los factores analizados para disminuir la mortalidad materna se acepta que los programas educativos que llegan a la comunidad en general y a la obstétrica en particular y el aumento en la cantidad y calidad de los especialistas han jugado un papel importante en dicho descenso.

Otro factor influyente ha sido el adelanto de la anestesia obstétrica.

La última revisión de mortalidad materna de nuestro Departamento fue elaborada en 1962 por Márquez (5).

Teniendo en cuenta la importancia que dicha mortalidad representa para una mejor adecuación de la atención obstétrica decidimos revisar las historias de las muertes ocurridas desde 1963 hasta 1972.

\section{Objetivos específicos:}

2.1. Analizar las causas directas, indirectas y no relacionadas de muertes maternas ocurridas en el Departamento de Obstetricia y Ginecología desde 1963 hasta 1972.

* Profesor 1 .

** Residente 11. - Depto. de Obstetricia y Ginecología. 
2.2. Comparar las causas de muertes en los dos períodos: 1963 a 1967 y 1968 a 1972.

\section{Material y Métodos:}

Aceptamos como muerte materna aquella que ocurre por cualquier causa durante el embarazo, parto y los 90 días siguientes a la terminación del embarazo, independiente de la duración y del sitio del mismo, por 10.000 nacidos vivos (1).

A - Muerte por causa directa: es aquella que resulta de cualquier complicación del embarazo, parto o puerperio y que fue resultado de una serie de hechos que se iniciaron por una complicación o una intervención.

B - Muerte por causa indirecta: la muerte es debida a una enfermedad desarrollada antes o durante el embarazo, la cual fue agravada por los efectos fisiológicos del mismo, pero no por causa directa del embarazo.

C - Muerte por causa no relacionada: cuando la muerte ocurre durante el embarazo, parto o puerperio por causas no relacionadas con el embarazo, ni con sus complicaciones 0 manejo.

Durante los diez años analizados se atendieron 40.680 partos y el número de nacidos vivos fue de 39.323 .

Se revisaron las 104 historias de muertes maternas ocurridas en el Departamento de Obstetricia y Ginecología, Hospital Universitaroi San Vicente de Paúl desde 1963 a 1972 y se dividieron en dos quinquenios.

Las variables analizadas fueron: procedencia, estadoc ivil, edad de la paciente, semanas de gestación, número de hijos, número de consultas prenatales, causas de muerte según el manual de la Asociación Médica Americana (1) y el estado final del feto.
Para la presente revisión se calculó la edad gestacional en semanas, en base a la fecha de la última menstruación.

En la paridad se anotaron solamente los partos o las cesáreas de las gestaciones a término.

En 50 historias se encontró el resultado del estudio anatomopatológico; cuando no se hizo dicho estudio, las historias fueron cuidadosamente analizadas y discutidas para determinar las causas directas y asociadas de la muerte.

Se utilizó una codificación manual y las causas de muerte fueron clasificadas según el listado aceptado por la Organización Mundial de la Salud (O.M.S.).

\section{Resultados:}

4.1. Procedencia. En los dos períodos, el lugar de procedencia fue igual, ya que el $50 \%$ correspondió a otros Municipios diferentes a Medellín. Las distancias entre estas localidades y la capital del Departamento, sumado al estado general de las pacientes al ingreso son factores que inciden en el número de muertes.

4.2. Estado Civil. La gran mayoría de las pacientes que ingresan a nuestro Departamento son casadas y esto se refleja lógicamente en los datos obtenidos.

4.3. Edad. La tercera parte de las pacientes del primer período eran menores de 30 años; mientras que en el segundo corresponden a este grupo etario las dos terceras partes.

4.4. Paridad. Cuarenta y cuatro pacientes $(42.3 \%)$ tenían menos de tres hijos. Treinta y cuatro pacientes $(32.6 \%)$ eran grandes multíparas y las restantes tenían entre cuatro $y$ seis hijos. 
4.5. Semanas de gestación. La edad gestacional fluctuó entre cuatro y cuarenta y dos semanas y el promedio fue de 16.15. Solamente en 4 pacientes no se conoció las semanas de gestación.

El número de abortos y la edad de estos embarazos son causantes de esta cifra promedio. Veintisiete pacientes ingresaron en post-parto y once en post-aborto.

4.6. Consulta Frenatal y estado final del feto. Durante el primer período solamente dos pacientes $1.9 \%$ habian asistido a consulta prenatal y en ambas el feto vivió. En las restantes sin consulta prenatal el feto murió en 33 casos. Durante el segundo período nueve pacientes $(8.65 \%)$ asistieron a la consulta prenatal y en seis de ellas el feto terminó en buenas condiciones. En las restantes 45 sin consulta prenatal, el feto murió en 26 de ellas.

4.7. Infección. (Cuadro № 1). Durante el primer período (1963-1967) se murieron 21 pacientes por sepsis sobre un total de 50 , esto represen-

\section{CUADRO NN 1}

\section{INFECCION}

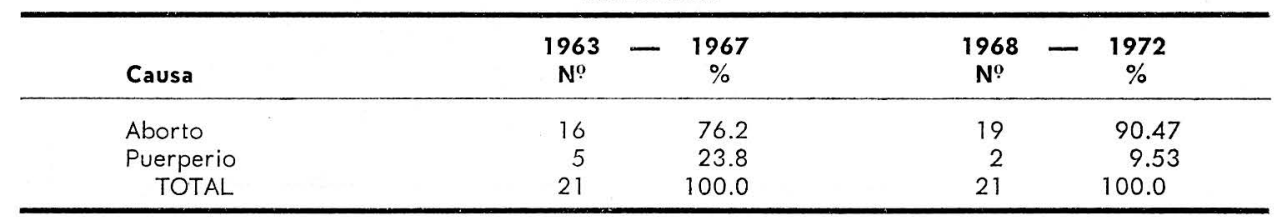

ta un $42 \%$ De las muertes por infección durante este tiempo 16 $(72.6 \%)$ fueron por aborto y solamente dos de estas pacientes confesaron maniobras abortivas. Durante el segundo período (1968-1972) se murieron 54 pacientes y 21 de ellas se debieron a la sepsis $(38.8 \%)$, correspondiendo al aborto 19 de esas muertes (90.47\%), observándose un aumento apreciable en relación con el primer período.
4.8. Hemorragia. (Cuadro № 2). La hemorragia fue la causa de la muerte en 18 pacientes durante el primer período (36\%). Al buscar la causa que desencadenó la hemorragia se aprecia que la coagulopatía, la placenta previa y el aborto fueron culpables de 4 casos cada una; sigue nla ruptura uterina con 3 ; el desprendimiento prematuro de la placenta con 2 y el embarazo ectópico en 1 caso.

\section{CUADRO N: 2}

\section{HEMORRAGIA}

\begin{tabular}{|c|c|c|c|}
\hline Causa & $1963-1967$ & $1968-1972$ & Total \\
\hline PLACENTA ACRETA & - & 2 & 2 \\
\hline COAGULOPATIA & 4 & - & 4 \\
\hline PLACENTA PREVIA & 4 & 2 & 6 \\
\hline ABORTO & 4 & 1 & 5 \\
\hline RETENCION DE PLACENTA & - & $i$ & 1 \\
\hline RUPTURA UTERINA & 3 & 2 & 5 \\
\hline DESP. PREMATURO DE PLACENTA & 2 & - & 2 \\
\hline EMB. ECTOPICO & 1 & - & 1 \\
\hline TOTAL & 18 & 8 & 26 \\
\hline
\end{tabular}


Durante el segundo quinquenio de estudio la hemorragia disminuyó a la mitad, solamente se presentaron 8 casos sobre 54 muertes (14.81\%). La placenta previa, la ruptura uterina y la placenta acreta fueron culpables de dos muertes cada una, en un caso fue el aborto y en otro la retención de placenta.
4.9. Toxemia. (Cuadro No 3). A diferencia de la hemorragia, la toxemia fue dos veces más frecuente en el segundo período. Se encontraron 14 casos sobre 54 muertes, lo cual representa $25.92 \%$; mientras que en el primer período se murieron 6 pacientes sobre un total de $50(12 \%)$.

\section{CUADRO Nํ 3}

\section{TOXEMIA}

\begin{tabular}{|c|c|c|c|}
\hline Causa & $1963-1967$ & $1968-1972$ & Total \\
\hline INSUFICIENCIA RENAL AGUDA & 1 & - & 1 \\
\hline HEMORRAGIA SUBARAC. & 1 & - & 1 \\
\hline INFECCION & 4 & 1 & 5 \\
\hline PARO CARDIACO & - & $i$ & 1 \\
\hline COLITIS ULCERATIVA & - & 1 & 1 \\
\hline ACC. CEREBROVASCULAR & - & 1 & 1 \\
\hline LEUCEMIA & - & 1 & 1 \\
\hline DESP. PREMATURO DE PLACENTA & - & 2 & 2 \\
\hline TOXEMIA & - & 7 & 7 \\
\hline TOTAL & 6 & 14 & 20 \\
\hline
\end{tabular}

Durante este primer quinquenio la insuficiencia renal aguda y la hemorragia subaracnoidea complicaron la toxemia en un caso cada una, mientras que la infección estuvo presente en cuatro casos.

Durante el segundo período la toxemia estuvo complicada por paro cardíaco de tipo anestésico, colitis ulcerativa, accidente cerebrovascular, leucemia y sepsis en un caso cada una y en dos se acompañó de desprendimiento prematuro de la placenta.
4.10. Causas indirectas. (Cuadro № 4). Tres pacientes murieron durante el primer período por causas indirectas y fueron debidas a neumonía lobar, insuficiencia cardíaca y hepatitis. Durante el segundo período se encontraron 4 causas indirectas de muerte, dos de ellas por hemorragia subaracnoidea y las otras por insuficiencia cardíaca y anemia por hemoglobina SC.

4.11. Causas no relacionadas. (Cuadro No 5). De las 104 muertes, 9

\section{CUADRO Nং 4}

\section{INDIRECTAS}

\begin{tabular}{|c|c|c|c|}
\hline Causa & $1963-1967$ & $1968-1972$ & Total \\
\hline NEUMONIA LOBAR & 1 & - & 1 \\
\hline INSUFICIENCIA CARDIACA & 1 & 1 & 2 \\
\hline HEPATITIS & 1 & - & $\overline{1}$ \\
\hline HEM. SUBARACN. & - & 2 & 2 \\
\hline ANEMIA HB. S/C & - & 1 & 1 \\
\hline TOTAL & 3 & 4 & 7 \\
\hline
\end{tabular}




\section{CUADRO N: 5}

NO RELACIONADAS

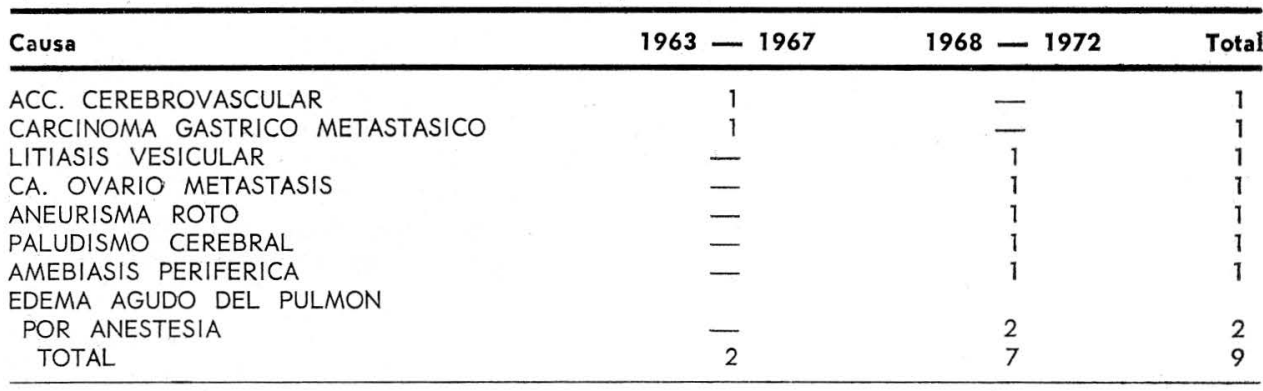

$(8.66 \%)$ fueron debidas a causas no relacionadas tales como: hemorragia cerebral por hipertensión arterial, metástasis de carcinoma gástrico, litiasis vesicular, metástasis de carcinoma de ovario, edema agudo del pulmón por accidente anestésico, aneurisma roto de aorta abdominal, amebiasis perforada y paludismo cerebral.
4.12. Causas de muerte por períodos. (Cuadro № 6). La infección ocupó el primer lugar como causa de muerte en los dos períodos: 42 y $38.8 \%$ respectivamente.

La hemorragia ocupó el segundo lugar en el primer período (36\%), mientras que el segundo período fue por toxemia ( $25.92 \%)$.

\section{CUADRO N: 6}

CAUSAS DE MUERTE POR PERIOdOS

\begin{tabular}{|c|c|c|c|c|}
\hline Causa & $\begin{array}{c}1963 \\
\mathrm{~N}^{\circ}\end{array}$ & $\begin{array}{c}1967 \\
\%\end{array}$ & $\begin{array}{c}1968 \\
\mathrm{~N}^{0}\end{array}$ & $\begin{array}{c}1972 \\
\%\end{array}$ \\
\hline Infección & 21 & 42 & 21 & 38.8 \\
\hline Hemorragia & 18 & 36 & 8 & 14.8 \\
\hline Toxemia & 6 & 12 & 14 & 25.9 \\
\hline No relacionadas & 2 & 4 & 7 & 12.9 \\
\hline Indirectas & 3 & 6 & 4 & 7.6 \\
\hline TOTAL & 50 & 100.0 & 54 & 100.0 \\
\hline
\end{tabular}

4.13. Causas globales de muerte. (Cuadro No 17). La infección ocupó el primer lugar y fue la causa del $40.38 \%$ de las muertes, el segundo lugar estuvo la hemorragia $25.0 \%$, el tercero la toxemia $19.23 \%$, luego las causas no relacionadas con $8.66 \%$ y por último las causas indirectas con $6.73 \%$.

4.14. Tasa de mortalidad materna. La tasa global de mortalidad materna

\section{CUADRO № 7}

CAUSAS GLOBALES

\begin{tabular}{lrr}
\hline Causa & \multicolumn{1}{c}{ No $^{\mathbf{0}}$} & $\%$ \\
\hline Infección & 42 & 40.0 \\
Hemorragia & 26 & 25.0 \\
Toxemia & 20 & 19.3 \\
No relacionadas & 9 & 8.6 \\
Indirectas & 7 & 6.7 \\
\multicolumn{1}{c}{ TOTAL } & 104 & 100.0 \\
\hline
\end{tabular}


fue de 26.40 por 10.000 nacidos vivos.

Al analizar la mortalidad materna por años encontramos que en 1962 la tasa fue de $5.7 \times 10.000$ y en 1965 de $61.0 \times 10.000$. No estamos en capacidad de explicar los factores que influyeron para estos hechos.

\section{Comentarios:}

En nuestro medio continúan ocupando los primeros lugares las causas clásicas de muerte en Obstetricia, como son la infección, la hemorragia y la toxemia.

Nuestro centro asistencial se ha convertido en un hospital de alto riesgo donde ingresan las pacientes con graves complicaciones enviadas de diferentes Municipios del Departamento.

El número de camas obsttéricas ha disminuído, especialmente por falta de recursos, y en la actualidad contamos con 59 para atender la población obstétrica y sus complicaciones tanto del Departamento de Antioquia como de Departamentos vecinos como Chocó y Córdoba.

Las pacientes jóvenes y con menos de tres hijos ocuparon el primer lugar entre las fallecidas, esto puede estar relacionado con el escaso número de embarazadas que asistieron a la consulta prenatal.

El aborto ocurrió en 40 casos $(38.46 \%)$ y fue causa de muerte por la hemorragia o por la infección. Nuestras pacientes jóvenes continúan pagando tributo a este terrible flagelo. En el Hospital de Gineco-Obstetricia No 3 del I.M.S.S. de México la mortalidad por aborto fue de $57.57 \%$ (6).

La tasa global de mortalidad materna por 10.000 nacidos vivos de
26.44 es muy elevada comparada con la de otros hospitales como el de Barquisimeto (Venezuela) que es de 10.3 (6) o el Rey Eduardo VIII de la U. de Natal (Africa) que es de 17 (4).

Mientras en otros paises se encuentra una disminución significativa de las muertes por infección, hemorragia y toxemia, entre nosotros continúan como causas principales con un aumento de la toxemia en el segundo período debido a una escasa y deficiente atención prenatal.

Es posible que el número de pacientes que ingresaron en malas condiciones después del parto o del aborto $(36.5 \%)$ estén jugando un papel significante en el número total de muertes.

El número de autopsias practicadas, $50(48 \%)$ es bajo en comparación con el de Barquisimeto ya citado (5) que fue de $95.73 \%$. El número de muertes debidas a la anestesia es muy bajo, si lo comparamos con otros estudios.

El análisis crítico de nuestros propios errores nos permitirá un examen detenido de los procedimientos futuros, encaminados a mejorar la atención materno-fetal.

\section{Resumen:}

Se analiza la mortalidad materna en el Hospital Universitario San Vicente de Paúl durante los años 1963 a 1967 y 1968 a 1972 y se comparan los resultados de los dos períodos.

Un total de 104 muertes se presentaron durante los 10 años y esto representa una tasa de 26.44 por 10.000 nacidos vivos.

Las causas de mortalidad materna fueron las siguientes: por infección $40.38 \%$, por hemorragia $25 \%$, por 
toxemia $19.23 \%$, por causas indirectas $6.73 \%$ y $8.66 \%$ por causas no relacionadas. La toxemia ocupó el tercer lugar como causa de muerte, pero comparando los dos períodos, llama la atención que fue dos veces más frecuente en el segundo (19681972), esto puede estar relacionado con una consulta prenatal deficiente en cantidad y calidad, sólo el 17\% de las pacientes habían asistido a dicha consulta y una mínima proporción de ella tuvo más de tres controles.

La hemoragia fue dos veces menos en el segundo período y podría explicarse por un mejor manejo de las complicaciones hemorrágicas del embaarzo, parto y puerperio.

La edad promedio fue de 30 años, con edades extremas entre 15 y 45.

La mitad de los casos procedían de otras poblaciones diferentes a Medellín y la mayoría de estas pacientes ingresaron en pésimas condiciones; podría así explicarse la alta tasa de mortalidad materna y es de anotar que fue ligeramente mayor en el segundo período: $27 \times 10.000$ nacidos vivos.

\section{Conclusiones:}

7.1. La tasa de mortalidad materna en el Hospital Universitario San Vicente de Paúl fue de 26.44 durante los años de 1963 a 1972.

7.2. La infección fue la principal causa de muerte $(40.38 \%$ ) y el aborto infectado fue su principal desencadenante.

7.3. La mayoría de estas muertes son prevenibles con una adecuada atención prenatal, un mejor manejo de las complicaciones del parto y puerperio y con campañas educativas.
7.4. Es muy importante el estudio piramidal y continuo de la mortalidad materna en los hospitales universitarios, que con criterios uniformes pueda ser utilizada para la enseñanza de atención materno-infantil.

7.5. Es deber del Gobierno adecuar los centros hospitalarios de recursos suficientes para atender la población obstétrica, especialmente la de alto riesgo.

\section{Summary:}

The Maternal Mortality in Hospital Universitario San Vicente de Paul during the years of 1963 to 1967 and 1968 to 1972 the results of the two periods are analysed and compared.

A total of 104 deaths occur during the 10 years, which represents a tasa of 26.44 per 10.000 borned alive.

The causes of Maternal Mortality were the following: By Infection $40.38 \%$, by Hemorrhage $25 \%$, by Toxemia $19.23 \%$ by Indirect Causes $6.73 \%$ and $8.66 \%$ by non relationed causes. The Toxemia occupied the third place as a cause of dead, but comparing the two periods, it caused the attention that was twice more frequent in the second one (19681972 ) this can be relationed with a deficient Prenatal Consult in quantity and quality, only the $17 \%$ of the patients had assisted to this consult and a minimum proportion of it had more than 3 controls.

Hemorrhage was twice less frequent during the second period and this could be explained by a better management of Hemorrhagyc Compli. cations of Pregnancy, Birth and Puerperium.

The mean age was 30 years, with extreme ages between 15 and 45 . Half of the cases came from other towns 
out of Medellin and the mayority of this patients were in bad conditions when they came in to the Hospital, thus explaining the high mortality rate, which was slightly higer during the second period: $27 \times 10.000$ borned alive.

\section{Conclusion:}

7.1. Mother Mortality rate during 1963 to 1972 at the Hospital Universitario San Vicente de Paul was of 26.44 .

7.2. Infection was the principal cause of dealth $(40.38 \%)$ and Septic Abortion was the principal desencadenating event.

7.3. The mayority of these deaths were Preventable with an Adecuate Prenatal Attention, better management of complications during birth and puerperium and education of campaigns.

7.4. A pyramidal and continuous study of Maternal Mortality in Universitary Hospital is very important since it can be used, with uniform criterial for the teaching of maternal new born attention.

7.5. It is a Government's duty to adecuate Hospitalary Centers with su- ficient measures to give obstetrical attention specially to High Risk Cases.

\section{BIBLIOGRAFIA}

1 Commitee on Maternal and Child Care of the A.M.A. "A guide for maternal death studies" 1964.

2 CRAWFORD, J. S. "The Anaesthetist's contribution to maternal Mortality". Brit. J. Anaesth. 40: 70-73, 1970.

3 CRESPO, J. "Mortalidad Materna en el Hospital Antonio María Pineda". Barquisimeto, Ven. 1959-1968. Rev. Gin. Obst. de Ven. 30 : 497-504. Septiembre 1970.

4 CRICHTON, D., KNOBEL, J. "Principles of Prfevention of Avoidable Maternal death: Study of 538 consecutive maternal deaths in the obstetric unit King Edward VIII Hospital". Darban 1953-1971 South Afr. Med. J. 47: 2005-1010 Oct. 271973.

5 MARQUEZ, L. "Mortalidad Materna en el Hospital Universitario San Vicente de Paúl". Mimeografiado 1962.

6 PEREZ de S., L., RUIZ, V. y ZETINA, F. "Mortalidad por Infección en el Hospital de Gineco-Obstetricia No 3 I.M.S.S. Gin. Obst. Mex. 29: 483-490 Mayo 1971.

7 RICE PUFFER, R., WYNNE GRIFFITH, G. "Características de la Mortalidad Urbana". Informe de la Investigación Interamericana de Mortalidad. Publicación No 151 O.M.S. Pág. 191 Mayo 1968. 